\section{Neumonía por Pneumocystis jiroveci post inicio de tratamiento con infliximab en paciente con enfermedad de Crohn}

\section{Daniela Fluxá, Jorge Segovia, Matías Florenzano, Jessica Salinas, Lilian Flores, Daniela Simian y Rodrigo Quera}

\section{Pneumocystis jiroveci pneumonia following infliximab therapy in patient with Crohn's disease}

There are no evidence-based guidelines about prophylaxis against Pneumocystis jiroveci pneumonia in inflammatory bowel disease. We report a case of $P$. jiroveci pneumonia in patient with Crohn's disease receiving infliximab and methotrexate. This case emphasizes the importance of considering the possibility of this infection in inflammatory bowel disease patients treated on biological therapy.

Key words: Pneumocystis jiroveci, Pneumocystis carinii, inflammatory bowel disease, Crohn's colitis, ulcerative colitis.

Palabras clave: Pneumocystis jiroveci, Pneumocystis carinii, enfermedad inflamatoria intestinal, enfermedad de Crohn, colitis ulcerosa.

\section{Introducción}

Pacientes con enfermedad inflamatoria intestinal (EII) en tratamiento con inmunomoduladores y terapia biológica tienen mayor riesgo de presentar infecciones ${ }^{1}$. Casos de neumonía por Pneumocystis jiroveci en pacientes con EII han sido asociados con el uso de estas terapias ${ }^{2}$. Esta es la primera comunicación nacional de una neumonía por $P$. jiroveci en un paciente con enfermedad de Crohn (EC) tratado con infliximab y metotrexato. Con este caso deseamos poner en alerta sobre la necesidad de considerar este cuadro en el diagnóstico diferencial ante un compromiso respiratorio en pacientes con EII que estén en tratamiento con terapia biológica e inmunomoduladores.

\section{Caso clínico}

Mujer de 48 años con antecedente de EC ileocolónica fenotipo inflamatorio. Se inició terapia con infliximab en esquema de inducción de 5 $\mathrm{mg} / \mathrm{kg}$ (0-2-6 semanas) asociado a metotrexato $25 \mathrm{mg} / \mathrm{semanal}$ vía oral presentando una respuesta clínica con un índice de Harvey-Bradshaw de 0 . A las cuatro semanas de la $3^{\text {a }}$ dosis, refirió compromiso del estado general, temperatura axilar de $37,5^{\circ} \mathrm{C}$, tos y disnea, requiriendo oxígeno por

Clínica Las Condes, Santiago, Chile. Departamento de Gastroenterología, Programa Enfermedad Inflamatoria Intestinal (DF, LF, RQ). Departamento de Medicina Interna, Unidad de Infectología (JSe), Centro de Enfermedades Respiratorias (MF), Unidad de Inmunología (JSa).

Dirección Académica, Subdirección de Investigación (DS).

Conflicto de intereses: no existen.

Recibido: 10 de marzo de 2017 / Aceptado: 22 de septiembre de 2017

\section{Correspondencia a:}

Rodrigo Quera P.

rquera@clc.cl naricera. Se internó para estudio, donde destacaba un hemograma con un hematocrito $41,2 \%$ y una hemoglobina de $13,7 \mathrm{~g} / \mathrm{dl}$, leucocitos $6.600 \mathrm{~mm}^{3}$ con baciliformes 5\%, linfocitos 15\%, PCR $96 \mathrm{mg} / \mathrm{L}$. La radiografía de tórax mostró tenues opacidades centrales perihiliares bilaterales (Figura 1). Se solicitó una tomografía computada (TC) de tórax que informó la presencia de opacidades en vidrio esmerilado y pequeñas áreas de relleno alveolar peribronquiales distribuidas difusamente en ambos campos pulmonares, engrosamiento septal bibasal y parietal difuso del árbol traqueobronquial y adenopatías mediastínicas, hiliares y axilares (Figura 2). Se realizó un lavado bronquioalveolar en que se realizó un panel viral respiratorio Filmarray ${ }^{\circledR}$ (ADV, coronavirus, metapneumovirus, rinovirus/enterovirus, Flu A y B, paraFlu, VRS, Chlamydophila pneumoniae, Mycoplasma

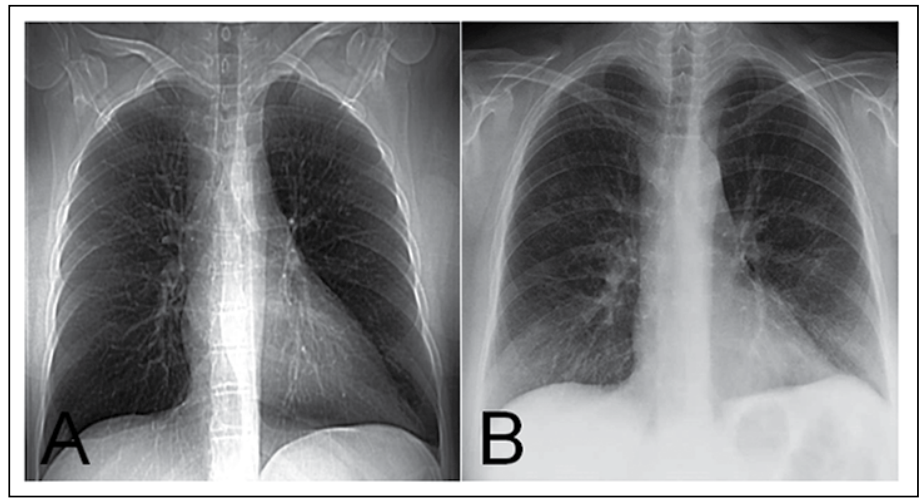

Figura 1. Radiografías de tórax normal previo al inicio de terapia con infliximab (A) y al momento del diagnóstico de la neumonía por Pneumocystis jiroveci. Se observan tenues áreas de mayor densidad perihiliares bilaterales que pueden corresponder a relleno alveolar (B).

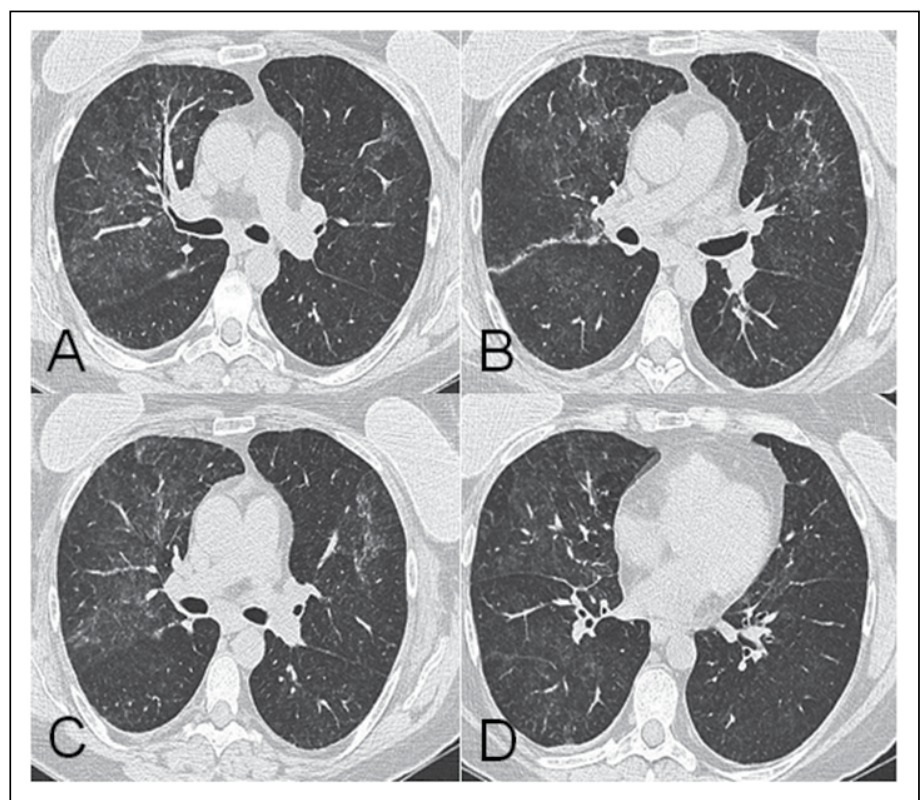

Figura 2. Tomografía computada de tórax que muestra alteraciones compatibles con proceso inflamatorio agudo. Se describen áreas con densidades en vidrio esmerilado y de relleno alveolar peribronquial. Engrosamiento parietal difuso del árbol traqueobronquial (A-D). 
pneumoniae y Bordetella pertussis), tinción de Giemsa y cultivo para bacterias y levaduras, RPC para Mycobacterium, baciloscopia y cultivo de Koch, resultando todos negativos. Los antígenos urinarios de Legionella y Streptococcus pneumoniae también fueron negativos. El estudio de RPC (técnica RPCq en tiempo real) en lavado bronquioalveolar para $P$. jiroveci fue positivo, iniciándose tratamiento con cotrimoxazol y corticoesteroides i.v. por 21 días. El recuento de linfocitos T CD4 por citometría de flujo fue de 219 céls $/ \mathrm{mm}^{3}$. La paciente evolucionó en forma favorable con regresión completa de sus síntomas respiratorios, manteniéndose la terapia combinada (infliximab y metotrexato) y profilaxis con cotrimoxazol oral, tres veces a la semana. El recuento de linfocitos T CD4 previo a la $5^{\mathrm{a}}$ y $6^{\mathrm{a}}$ dosis fue de 848 céls $/ \mathrm{mm}^{3}$ y 922 céls $/ \mathrm{mm}^{3}$; respectivamente.

\section{Discusión}

El riesgo de neumonía por $P$. jiroveci en pacientes con EII es extremadamente bajo alcanzando menos de 0,3 casos por 100 personas/año expuestos a tratamiento con corticoesteroides, inmunosupresores o terapia biológica ${ }^{3}$.

En cuanto al hallazgo de $P$. jiroveci en la vía respiratoria es importante considerar que hasta $16 \%$ de los pacientes con enfermedades autoinmunes pueden estar colonizados ${ }^{4}$, por lo que su presencia en forma aislada y sin un contexto clínico adecuado, deben ser interpretados con precaución.

Dado que el estudio microscópico del $P$. jiroveci sólo ha mostrado una sensibilidad de un $55-78 \%$, han sido desarrolladas nuevas herramientas diagnósticas ${ }^{5}$. En este sentido técnicas moleculares de RPC y serológicas que evalúan el polisacárido $\beta$-D-glucano pueden ayudar en el diagnóstico y manejo de la neumonía por $P$. jiroveci ${ }^{5}$. Sin embargo, es necesario considerar que, aunque la técnica de RPCq es promisoria en discriminar colonización e infección, las secuencias de ADN a evaluar y el valor de corte aún deben ser estandarizadas.

Múltiples factores de riesgo se han descrito para el desarrollo de neumonía por $P$. jiroveci en pacientes con EII. El uso de corticoesteroides es uno de los factores de riesgo más importante, presente en 50 a $65 \%$ de los casos de neumonía por $P$. jiroveci en población no- $\mathrm{VIH}^{6}$. Su mecanismo estaría relacionado con alteraciones en la función de los macrófagos/ monocitos y de los LT. Este riesgo varía según la dosis y duración del uso de los corticoesteroides, siendo mayor en los primeros seis meses de tratamiento, similar a lo que ocurre con la terapia biológica anti-TNF${ }^{7}$.

Distintos mecanismos han sido sugeridos para explicar el rol de infliximab en el desarrollo de la neumonía por $P$. jiroveci. Los agentes anti-TNF causan un deterioro de la inmunidad celular, humoral y disminución de los recuentos de los linfocitos T CD4. Por otra parte, estudios in vitro han demostrado que la secreción de TNF por los macrófagos alveolares sería un mecanismo de defensa contra $P$. jiroveci, con una mayor producción de TNF asociada a menores recuentos de $P$. jirovec $i^{8}$.
En conclusión, nuestro caso demuestra que con el mayor uso de agentes anti-TNF en pacientes con EII, es necesario considerar la neumonía por $P$. jiroveci dentro de las causas de infecciones oportunistas en este grupo de pacientes ${ }^{1}$. La evaluación de factores de riesgo como el estado nutricional, patología respiratoria concomitante, hospitalización reciente por EII, recuento de linfocitos y terapia inmunosupresora asociada ${ }^{6,7,9,10}$ permitirán determinar en qué escenario será necesario utilizar profilaxis realizando de esta manera un manejo individualizado ${ }^{6-11}$.

\section{Referencias bibliográficas}

1.- Hutfless S M, Weng X, Liu L, Allison J, Herrinton L J. Mortality by medication use among patients with inflammatory bowel disease, 19962003. Gastroenterology 2007; 133: 1779-86.

2.- Sharma K, Rao P, Krishnamurthy P, Ali S A, Beck G. Pneumocystis carinii jiroveci pneumonia following infliximab infusion for Crohn's disease: emphasis in prophylaxis. South Med J 2007; 100: 331-2.

3.- Cotter T G, Gathaiya N, Catania J, Loftus E V Jr, Tremaine W J, Baddour $\mathrm{L}$ M, et al. Low risk of pneumonia from Pneumocystis jirovecii infection in patients with inflammatory bowel disease receiving immune suppression. Clin Gastroenterol Hepatol 2017; 15: 850-6.

4.- Mekinian A, Durand-Joly I, Hatron P Y, Moranne O, Denis G, Dei-Cas $\mathrm{E}$, et al. Pneumocystis jirovecii colonization in patients with systemic autoimmune diseases: prevalence, risk factors of colonization and outcome. Rheumatology (Oxford) 2011; 50: 569-77.

5.- White P L, Backx M, Barnes R A. Diagnosis and management of Pneumocystis jirovecii infection. Expert Rev Anti Infect Ther 2017; 15: 435-47.

6.- Long M D, Farraye F A, Okafor P N, Martin C, Sandler R S, Kappelman M D. Increased risk of Pneumocystis jiroveci pneumonia among patients with inflammatory bowel disease. Inflamm Bowel Dis 2013; 19:1018-24.

7.- Lawrance I C, Radford-Smith G L, Bampton P A, Andrews J M, Tan P $\mathrm{K}, \mathrm{Croft} \mathrm{A}$, et al. Serious infections in patients with inflammatory bowel disease receiving anti-tumor-necrosis-factor-alpha therapy: an Australian and New Zealand experience. J Gastroenterol Hepatol 2010; 25: 1732-8.

8.- Krishnan V L, Meager A, Mitchell D M, Pinching A J. Alveolar macrophages in AIDS patients: increased spontaneous tumor necrosis factor-alpha production in Pneumocystis carinii pneumonia. Clin Exp Immunol 1990; 80: 156-160.

9.- Okafor P N, Nunes D P, Farraye F A. Pneumocystis jiroveci pneumonia in inflammatory bowel disease: when should prophylaxis be considered? Inflamm Bowel Dis 2013; 19: 1764-71.

10.- Viget N, Vernier-Massouille G, Salmon-Ceron D, Yazdanpanah Y, Colombel J F. Opportunistic infections in patients with inflammatory bowel disease prevention and diagnosis. Gut 2008; 57: 549-58.

11.- Rahier J F, Magro F, Abreu C, Armuzzi A, Ben-Horin S, Chowers Y, et al. Second European evidence-based consensus on the prevention, diagnosis and management of opportunistic infections in inflammatory bowel disease. J Crohns Colitis 2014; 8: 443-68. 